\title{
Duration of untreated illness and outcome in schizophrenia: test of predictions in relation to relapse risk
}

David Cunningham Owens, Eve C. Johnstone, Patrick Miller, J. Fiona Macmillan and Timothy J. Crow

\section{Background}

The nature of the relationship between duration of the prediagnostic interval in schizophrenia and better outcomes remains unclear.

\section{Aims \\ To re-examine data from one of the earliest studies suggesting an association between long pre-treatment interval and compromised outcome, assessing the relationship between symptomatic and social variables and increased relapse risk at 1 year.}

\section{Method}

Symptomatic, social and demographic data from participants in the Northwick Park Study of First Episodes who completed 12-month follow-up $(n=101)$ were re-analysed in the context of duration of untreated illness (DUI).

\section{Results}

At admission, those with long DUI were more likely to have lower scores on tension derived from the Present State Examination, exhibited more behaviour threatening to others and more bizarre behaviour, were more likely to be single, to live alone or dependently, to be unemployed and to have experienced more adverse life events prior to admission. Logistic regression showed that diminished tension, bizarre behaviour and unemployed status independently increased the risk of relapse, bizarre behaviour making the single biggest contribution. Tension did not remain significant with log-transformation of data.

\section{Conclusions}

Findings are consistent with the conclusion that long DUI can reflect characteristics of the psychosis itself rather than delay in treatment.

\section{Declaration of interest}

None.
Reduction in the time over which a psychotic process evolves from emergence of the first features of disorder to diagnosis and instigation of treatment (DUI, duration of untreated illness/ DUP, duration of untreated psychosis) underpins early intervention. Considerable impetus to development in this field came from an influential review by Wyatt ${ }^{1}$ in which he argued that patients with schizophrenia who received antipsychotics earlier had better long-term outcomes. Two reviews agree that shorter DUP is associated with modest benefits in short-term outcomes across a number of domains. ${ }^{2,3}$ This does not however establish a direct causative relationship, ${ }^{2}$ with individual results still open to interpretation. ${ }^{4,5}$ The question of whether the association is confounded by some other factor(s) inherent in different 'types' of illness remains. ${ }^{2,6}$ Furthermore, although a range of outcomes have been assessed, including symptoms and syndrome dimensions, psychosocial functioning/quality of life and cognition, with variable results, few studies have looked at relapse, results with this outcome again being contradictory. ${ }^{3}$

One of the earliest sources from which Wyatt drew evidence was the Northwick Park Study of First Episodes, designed to assess the value of maintenance antipsychotics following first schizophrenia episodes. ${ }^{7}$ This found a correlation between prolonged DUI and shortened time to relapse. Two possible explanations were offered - that some features associated with high relapse risk lead to delay in admission (e.g. insidious onset which might independently be associated with poor outcome; poor social support which might also be related to early relapse following discharge) or, the 'more arresting explanation', that delay in instituting treatment itself leads to poorer outcome. The authors speculated that 'the presence of symptoms untreated by neuroleptic drugs leads to abnormality which cannot be completely reversed by subsequent treatment', adding 'such a possibility should not be too readily dismissed.7 This was the first time these two possibilities were articulated and far from being dismissed, the second was the one enthusiastically pursued. In view of the importance of these conclusions to an ongoing debate, we have returned to the original data to address which of these two proposals - the deleterious effect of treatment delay itself or the presence of factors(s) that relate to both delayed presentation and high risk of relapse - best accounts for the fact that within this population there was a group of patients with schizophrenia with prolonged symptomatology prior to treatment who, after recovery, relapsed more rapidly than those with shorter pre-treatment intervals. We hypothesised that longer pre-treatment interval would be associated with: (a) more severe illness at presentation; and (b) the presence of features that independently relate to increased risk of relapse.

\section{Method}

The Northwick Park Study of First Episodes has been extensively described elsewhere. ${ }^{7-9}$ In summary, 462 patients were referred from nine medical centres within a 35-mile radius of the London Borough of Harrow for assessment as possible first-episode cases of schizophrenia. Of these, 235 conformed to study criteria, 120 of whom recovered from their initial episode to enter a randomised, placebo-controlled trial of antipsychotic medication to assess the efficacy of maintenance therapy. The present sample comprises 101 individuals with adequate information who completed the 12 -month period following entry.

Assessments at entry included the Present State Examination (PSE), ${ }^{10}$ the World Health Organization (WHO) Past History and Sociodemographic Schedule (PHSD) and Disability Assessment Schedule (DAS), ${ }^{11}$ and the Disturbed Behaviour Rating (DBR) 
based on relative/informant interview and devised to provide a standardised recording of objective behavioural difficulties. ${ }^{8}$ The DBR assesses five areas: behaviour threatening to the life of the patient; behaviour threatening the lives of others; inappropriate sexual behaviour; damage to property; and bizarre behaviour not otherwise specified (NOS). Behaviours were recorded as absent or present once, more than once, or repeatedly for more than 1 month. As the PSE provided the primary criteria for entry into the study and was performed first, clinical data were masked to knowledge of the duration of prior symptomatology.

The present analysis relates to DUI defined from the onset of any noteworthy symptomatology, not specifically psychotic phenomena, with onset being determined from data contained in the PHSD, the DAS and the DBR.

Recovery was clinically determined by the responsible clinical team, and following discharge, patients were reviewed at least once every 6 weeks until relapse/readmission or until 12 months after discharge.

For the primary analysis, the total sample was divided into three equal groups arbitrarily representing 'short' (Group B), 'intermediate' (Group C) and 'long' (Group A) pre-treatment intervals. There can be as yet no theoretical basis for such subdivisions, ${ }^{3}$ the device reflecting a statistical need to deal with skew in the data. The present analysis relates to those within these groups who completed 12 months in the study: Group A: onset of illness more than 1 year prior to admission $(n=28)$; Group B: onset of illness less than 44 days prior to admission $(n=29)$; Group C: remaining participants with onset of illness between 44 days and 1 year $(n=44)$.

Severity of the first episode of illness was assessed in terms of: symptomatology from admission PSE, expressed in terms of the composite scales derived by Owens et al; ${ }^{12}$ overall Index of Definition from the PSE (ranging from 1, indicative of no illness, to 8 , severe illness) ${ }^{10}$ duration of admission with the first episode of illness; and degree of behavioural disturbance prior to admission as described by third-party informants and recorded on the DBR.

Environmental or external factors shown to be associated with, or to be precursors of, onset/relapse ${ }^{13}$ for which data were available from the Northwick Park data-set include: social support variables (marital status, living environment, availability of confidant/e); employment status; life events prior to admission; illicit substance use, especially cannabis; ethnicity; limited facility with English; and familiality of the condition.

Although life events were recorded in detail on the WHO scales, they represented a diversity of circumstances. The present analysis is restricted to those events deemed to represent 'serious' intrusions - dismissal from job, major legal difficulties, death of significant other, divorce, substantive change in relationship with spouse/partner and suicide attempt.

Dichotomous variables were considered in terms of the chisquared test. Correlational analyses were undertaken for indices of illness severity and environmental/external factors relating to increased relapse risk, across the three DUI groups. Significant variables were entered into a logistic regression to construct a model for the prediction of relapse in this sample within 1 year.

\section{Results}

Differences in relapse rates in the three groups within 1 year are shown in Table 1. This confirms that those with a long 'lead' time from illness onset to start of antipsychotic treatment have a significantly higher relapse rate than those with short or intermediate DUI.

\section{Parameters of severity}

Differences in measures of severity (excluding behaviour) between the three groups are shown in Table 2. The only significant symptom difference in PSE-derived symptoms scales was that tension was significantly less severe in those with long lead times. The Index of Definition was close to maximum in all groups and did not significantly differ, and there was no significant difference in the time spent in hospital between the groups.

Findings for behavioural parameters are shown in Table 3. Those with longer lead times differed from the others in that 'behaviour threatening to others' $(P<0.03)$ and 'repeated bizarre behaviour NOS' $(P=0.003)$ were significantly more common.

\section{Social/environmental factors}

As shown in Table 4, on admission Group A individuals were more likely to be single with no partner $(P=0.005)$, to be living alone/with parents $(P=0.033)$ and were less likely to be employed $(P=0.001)$. Although less consistent, life events results suggested that those with long lead times were less likely than others to have experienced a major life event in the 3 months prior to admission. No significant differences emerged in terms of cannabis use (relatively uncommon at this time), family history of psychotic illness, ethnicity or possible language differences.

Thus, at admission to hospital, significant differences existed between the groups in terms of the symptomatic variables of tension, behaviour threatening to others, and bizarre behaviour NOS, and the social variables of marital status, living environment,

\begin{tabular}{|c|c|c|c|}
\hline & $\begin{array}{c}\text { Group A } \\
\text { (long), a } n \%\end{array}$ & $\begin{array}{c}\text { Group B } \\
\text { (short), }{ }^{\mathrm{b}} n \%\end{array}$ & $\begin{array}{c}\text { Group C } \\
\text { (intermediate), }{ }^{\mathrm{C}} n \%\end{array}$ \\
\hline No relapse within 1 year & $5(17.9)$ & $17(58.6)$ & $22(50.0)$ \\
\hline Relapse within 1 year & $23(82.1)$ & $12(41.4)$ & $22(50.0)$ \\
\hline \multicolumn{4}{|c|}{$\begin{array}{l}\chi^{2}=10.9, P=0.004 \text {. } \\
\text { a. Onset of illness more than } 1 \text { year prior to admission }(n=28) \text {. } \\
\text { b. Onset of illness less than } 44 \text { days prior to admission }(n=29) \text {. } \\
\text { c. Remaining participants with onset of illness between } 44 \text { days and } 1 \text { year }(n=44) \text {. }\end{array}$} \\
\hline
\end{tabular}

Table 2 Duration of untreated illness and markers of illness severity on admission

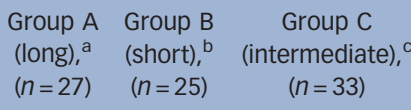

PSE-derived symptom scale score

\begin{tabular}{|c|c|c|c|}
\hline Situational anxiety & 0.52 & 0.44 & 0.79 \\
\hline Tension & 3.26 & 6.12 & $4.70 *$ \\
\hline Depression & 7.00 & 9.32 & 7.33 \\
\hline Mania & 0.37 & 0.32 & 0.18 \\
\hline Overactivity & 0.44 & 0.92 & 0.48 \\
\hline Incoherence & 0.81 & 1.16 & 0.94 \\
\hline Perceptual change & 1.96 & 2.52 & 1.76 \\
\hline Hallucinations & 4.59 & 4.40 & 5.64 \\
\hline Thought disturbance & 1.74 & 1.32 & 2.00 \\
\hline Delusional construction & 13.44 & 14.16 & 12.82 \\
\hline Outside control & 4.77 & 4.32 & 4.06 \\
\hline Negative symptoms & 1.00 & 0.60 & 1.18 \\
\hline dex of Definition (PSE) & 7.41 & 7.88 & 7.73 \\
\hline $\begin{array}{l}\text { ean duration of hospitalis } \\
\text { ays }\end{array}$ & 91.8 & 85.1 & 86.8 \\
\hline
\end{tabular}




\begin{tabular}{|c|c|c|c|c|}
\hline & Group A (long) ${ }^{a}$ & Group B (short) ${ }^{b}$ & Group C (intermediate) ${ }^{c}$ & $\chi^{2}$ \\
\hline Behaviour threatening to patient, $n(\%)$ & & & & 0.45 \\
\hline No & $20(76.9)$ & $20(69.0)$ & $28(73.7)$ & \\
\hline Yes & $6(23.1)$ & $9(31.0)$ & $10(26.3)$ & \\
\hline Behaviour threatening to others, $n$ (\%) & & & & $7.05, P=0.029$ \\
\hline No & $18(69 . \%)$ & $26(89.7)$ & 35 (92.1) & \\
\hline Yes & $8(30.8)$ & $3(10.3)$ & $3(7.9)$ & \\
\hline Disturbed sexual behaviour, $n$ (\%) & & & & 0.60 \\
\hline No & $24(92.3)$ & $25(86.2)$ & $33(86.8)$ & \\
\hline Yes & $2(7.7)$ & $4(13.8)$ & $5(13.2)$ & \\
\hline Damage to property, $n$ (\%) & & & & 4.79 \\
\hline No & $20(76.9)$ & $26(89.7)$ & $36(94.7)$ & \\
\hline Yes & $6(23.1)$ & $3(10.3)$ & $2(5.3)$ & \\
\hline Bizarre behaviour, $n$ (\%) & & & & $16.3, P=0.003$ \\
\hline No & $8(30.8)$ & $14(48.3)$ & $15(39.5)$ & \\
\hline \multicolumn{5}{|l|}{ Yes } \\
\hline Once or repeated over 1 month & $4(15.4)$ & $13(44.8)$ & $14(36.8)$ & \\
\hline Repeated over $>1$ month & $14(53.8)$ & $2(6.9)$ & $9(23.7)$ & \\
\hline
\end{tabular}

\begin{tabular}{|c|c|c|c|c|}
\hline & Group A (long) ${ }^{a}$ & Group B (short) ${ }^{b}$ & Group C (intermediate) ${ }^{\mathrm{c}}$ & $\chi^{2}$ \\
\hline Marital status, $n(\%)$ & & & & $14.7, P=0.005$ \\
\hline Never married & $18(69.2)$ & $14(51.9)$ & $28(66.7)$ & \\
\hline Married/partner & $3(11.5)$ & $13(48.1)$ & $7(16.7)$ & \\
\hline Separated/widowed/divorced & $5(19.2)$ & 0 & $7(16.7)$ & \\
\hline Living arrangements, $n$ (\%) & & & & $13.7, P=0.033$ \\
\hline Alone & $4(15.4)$ & 1 (3.6) & $3(7.1)$ & \\
\hline With spouse & $3(11.5)$ & $12(42.9)$ & $7(16.7)$ & \\
\hline With parent & $16(61.5)$ & $10(35.7)$ & $20(47.6)$ & \\
\hline Other & $3(11.5)$ & $5(17.9)$ & $12(28.6)$ & \\
\hline Employment status, $n$ (\%) & & & & $18.5, P=0.001$ \\
\hline Unemployed & $19(73.1)$ & $5(17.9)$ & $16(38.1)$ & \\
\hline Sick leave & $6(23.1)$ & $14(50.0)$ & $18(42.9)$ & \\
\hline Other & $1(3.8)$ & $9(32.1)$ & $8(19.0)$ & \\
\hline Life events, ${ }^{d} n(\%)$ & & & & $9.80, P=0.44$ \\
\hline None & $13(46.4)$ & $15(51.7)$ & $13(29.5)$ & \\
\hline At least one in the previous 3 months & $4(14.3)$ & $10(34.5)$ & $44(43.6)$ & \\
\hline At least one in the previous $4-12$ months & $11(39.3)$ & $4(13.8)$ & $13(29.5)$ & \\
\hline
\end{tabular}

employment status and life events. The relationship of each of these to relapse was then explored.

A lower tension score was significantly related to likelihood of relapse $(F=4.37, \quad P=0.04)$, as was the presence of bizarre behaviour NOS $(F=11.58, P=0.003)$ and unemployment on admission $(F=8.75, P=0.013)$. None of the other variables were significantly associated with relapse.

Using logistic regression, the best model for predicting relapse was constructed. The significant predictors were:
(a) a relative absence of tension at admission
(b) bizarre behaviour evident at least once over 1 month prior to admission
(c) repeated bizarre behaviour prior to admission
(d) being unemployed on discharge from hospital
(e) being assigned to placebo in the clinical trial.

Once variables (a) to (d) are entered into the model, lead time prior to hospitalisation does not make a significant contribution. None of the variables considered predicted time to relapse.

In addition to this primary analysis with the division of the cohort into three groups on the basis of DUI, a supplementary analysis was conducted where this variable was modelled as a continuous term. The DUI showed a positive skew and was transformed using the formula $\log ^{(x+1)}$ to avoid the appearance of missing data and to maintain the assumption of a linear relationship with the log-odds of relapse.

The results were similar to those seen with the primary analysis - a history of bizarre behaviour and trial treatment allocation remained significant predictors of relapse, although tension no longer maintained significance. In agreement with the previous analysis, time to treatment was not significantly associated with the subsequent likelihood of relapse. 


\section{Discussion}

\section{Validity of data}

The criticism that the present analysis represents a 'fishing expedition' with geriatric data must be addressed. The data-set was indeed compiled over a quarter of a century ago but comprises material collected by experienced researchers according to the exacting standards that prevailed for all research samples of Northwick Park Hospital at the time. The major instruments were meticulously developed, internationally validated, have aged well and would be acceptable in modern studies. The DBR was devised for the study and, as a simple means of categorising behavioural disturbance, has face validity. We would therefore stand by the continuing relevance of the raw data. The study concerned relapse, an outcome infrequently addressed in the literature on pre-treatment symptomatic intervals ${ }^{3}$ and the design allowed for clinical assessments at entry that were masked to illness evolution, a continuing issue in this field. Marshall et al ${ }^{2}$ identified only 2 out of their 26 studies that met this criterion. In addition, the endpoint (relapse) was also in effect determined masked - by the responsible clinicians not the research team, a practice principle that may strengthen older data. Although many options are now available for the treatment of those with psychotic illness, and various professionals may contribute to or decide on outcome parameters such as relapse, resulting in doubts about representativeness of study samples, this was not the case at the time this study was undertaken, when relapse was determined by a single, usually senior clinician and admission virtually universal. We would therefore also stand by the relevance of further analyses of these data.

\section{Defining the focus}

Two issues have the potential to blur clarity in the early intervention debate. Wyatt's review related specifically to schizophrenia but much research supporting early intervention as a means of reducing pre-treatment intervals concerns psychosis in general or schizophrenia-spectrum disorders. In Marshall et al's review, which attempted to restrict publications to those concerning 'schizophrenia-like disorders', 4 of the 26 studies reported data 'for all psychoses only'. Although a degree of diagnostic uncertainty is inevitable in early psychosis, it is important where possible to return the debate to its original terrain, as it is with schizophrenia that the longest delays in accessing treatment occur $^{14,15}$ and outcome benefits from reducing delays should be greatest if the association is direct. The data presented here relate specifically to schizophrenia, defined by PSE Catego criteria, i.e. to a narrow concept of the condition. ${ }^{10}$ In addition, however, the sample was followed up in detail at 2 years, ${ }^{16}$ which confirmed individual diagnoses. Participants would therefore conform to both DSM-IV ${ }^{17}$ and ICD-10 ${ }^{18}$ criteria for schizophrenia.

Second, a distinction between DUI (time from first manifestation of any features until adequate antipsychotic treatment) and DUP (time from first manifestation of any psychotic features to treatment) has become accepted, the literature overwhelmingly focusing on the latter. There is some support for maintaining the distinction. Barnes and colleagues found no correlation between the two, although some variables related differently to DUI as opposed to DUP, ${ }^{19,20}$ an observation evident in other work. ${ }^{21}$ Nonetheless, the rationale behind the distinction is weak. It is rarely stated whether DUP is adopted to reflect practicalities (namely, the relative reliability of identifying psychotic as opposed to 'non-specific' phenomena in retrospect), or whether the point is theoretical, accepting, as Wyatt proposed, that emergence of psychotic features reflects some 'toxic' biological process that either promotes the shift to full-blown schizophrenia or is stimulated by it. Prospective assessments have shown that the presence of partial or transient psychotic phenomena does not in itself predict progression to formal schizophrenic illness and hence, in familial cases at least, cannot per se reflect a threshold event, ${ }^{22}$ whereas non-specific symptomatology is virtually universal in those whose course is to illness. ${ }^{12}$ In those already diagnosed, awareness of emerging non-specific phenomena would seem a valid marker of the earliest change denoting the shift to illness. Our data refer to the emergence of features considered by close third parties, especially relatives, as of significance with regard to the subsequent development of illness and would correspond to DUI.

\section{Parameters of severity}

We found no evidence that a longer period prior to accessing treatment was associated with more severe illness at presentation in terms of global symptom levels. Although an association between longer DUP and higher global symptom ratings has been reported, ${ }^{23}$ our finding utilising a DUI measure is in line with the consensus using DUP. ${ }^{3}$ Our Index of Definition data support the interpretation of Barnes and colleagues, ${ }^{20}$ who attributed a lack of association in their study to the fact that service contact was triggered by patients reaching a severity threshold. Likewise, our groups did not differ in terms of duration of hospitalisation, a finding reported even where early detection services have been

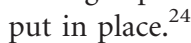

The sole symptomatic association we found was that those with a longer pre-treatment interval had lower scores on the PSE-derived tension scale at admission, which was also independently associated with relapse, although this relationship did not withstand the supplementary analysis. Tension is a composite of nine items: 4 (worrying), 5 (tension pains), 6 (tiredness), 7 (muscular tension), 8 (restlessness), 10 (feeling of nervous tension), 11 (free-floating anxiety), 12 (anxious foreboding with autonomic accompaniments) and 120 (observed anxiety). Psychopathologically, these represent a homogeneous set of phenomena reflecting anxiety/arousal in the most general sense, but as a construct, tension is not directly equatable with those embedded in standardised assessment instruments most commonly utilised in the modern literature, making comparisons difficult. In apparent contradiction of our finding, there is evidence that anxiety and depression at presentation are greater in those with long DUP and associated with poorer outcome at 6 and 12 months, ${ }^{2}$ but studies in general have not concentrated on this domain. The failure of the relationship between tension and relapse to be maintained following log-transformation of our data suggests that such non-specific symptomatology may not represent a robust predictor of outcome.

The most consistent symptomatic association in the literature is with negative symptoms, long DUP being associated with greater levels both at presentation and especially at follow-up. ${ }^{2,3}$ The present data, in relation to presentation, do not in general support this. The PSE-derived score for 'negative' symptoms comprises 6 items (110, slowness; 119, catatonic movements; 128, blunted affect; 130, slow speech; 133, muteness; and 134, restricted quantity of speech) all of which are observed signs, and although there is clear overlap with specific negative symptom rating scales, the constructs are not entirely consonant. Negative symptomatology continues to present profound conceptual and measurement problems for psychiatry ${ }^{25}$ in the light of which one must be cautious about the weight of interpretation one places upon its presence. Our findings, using a somewhat different appraisal method from the literature in general, support such caution.

Although it would be rash to assume that diminished tension and negative symptoms tap into the same domain, at a clinical 
level, both would be compatible with social disengagement that could underlie delayed access to services.

A further element to emerge from our data was the role of behavioural disturbance, both as a factor proximate to admission (and probably determining it), but also as an independent predictor of relapse. The literature does not in general consider behaviour as a separate domain of disorder, which is surprising as, clinically, behavioural disturbance is a frequent catalyst for admission and as such is an intuitive marker of severity. In addition, there is evidence from samples with chronic illness at least, that disturbance in the behavioural domain correlates with negative but not positive features. ${ }^{26}$ We found that those with longer pre-treatment intervals showed evidence of more disturbed behaviour, both threatening to others and of a bizarre nature, prior to admission, but it was the latter that independently increased relapse risk in both the primary and supplementary analyses. Repeated bizarre behaviour (not classifiable as threatening, sexual or damaging to property) was the strongest independent predictor of early relapse, even more so than being allocated to placebo. The presence of a rating for this reduced the odds of a favourable 1-year outcome by $90 \%$ if manifest repeatedly and by $85 \%$ if manifest only in the month prior to admission. We have no evidence that early relapse in such patients most likely reflects poor long-term adherence, although this is clinically intuitive, particularly in view of the standing of this behavioural variable in relation to placebo trial allocation.

The association between DUP and poor premorbid personality, an obvious candidate for exerting independent and adverse effects on outcome, remains controversial, although systematic review does not support this as confounding the relationship between DUP and outcome. ${ }^{2}$ There is, however, evidence that mode of onset may be a related factor of importance, those with a more rapid onset and shorter DUP having a better outcome. ${ }^{27,28}$ The chronicity of the behavioural disturbance evident within the Northwick Park sample prior to admission was often a striking feature of third-party accounts ${ }^{8}$ as were the high thresholds of tolerance that developed, notwithstanding the distress engendered. Such chronicity is characteristic of insidious onset and compatible with an impaired level of social and interpersonal relationships.

The picture of longer pre-treatment intervals being associated with gradual evolution is supported by the other significant findings relevant at time of admission - those with longer pretreatment intervals were more likely to be single, to be living alone/with parents (i.e. not in a stable partnership) and to be unemployed, and were less likely to have experienced a significant life event immediately prior to hospitalisation. Our data from a sample with schizophrenia did not support the finding ${ }^{21}$ that DUI is longer in those with a positive family history of psychotic illness. The logistic regression model providing the best predictors of early relapse included only tension, bizarre behaviour (both over 1 month and repeatedly), being unemployed and being treated with placebo. This model correctly predicted $54.8 \%$ of those who did not relapse within 1 year and $84.8 \%$ of those who did. The DUI was not significantly associated with relapse within 1 year and was removed from the model when these variables were entered.

\section{Heterogeneity and the direction of causality}

The design of the Northwick Park study does not allow us to determine the direction of causality, an issue that after a quarter of a century remains in much of the relevant literature. The present findings do, however, add to evidence that the relationship between pre-treatment interval, however defined, and outcomes in schizophrenia is unlikely to be straightforward, a view in line with Wyatt's very specific conclusion that 'early intervention with neuroleptics in some [author's emphasis] acute psychotic patients reduces long-term morbidity. ${ }^{1}$ Some contradictions in the literature most probably reflect heterogeneity, not only in methodologies $^{29}$ but across and within diagnostic categories. ${ }^{30}$ With regard to treatment response, it has been suggested that DUP exerts its impact on those 'with a less pernicious, more reversible form of illness. ${ }^{31}$ This leaves open the likelihood that in some patients the relationship is confounded by factors independently linking prolonged pre-treatment interval and poor outcome. In the present study, the nature of these variables encourages the view that early symptomatic, behavioural and social indicators of poor outcome would provide a valid focus for early intervention strategies, highlighting factors such as unemployment as targets for identifying those likely to engage poorly. ${ }^{32}$ However, as with much of this literature in which pretreatment interval was not the primary issue in design, our findings cannot address the issue conclusively but still leave open the alternative interpretation, the first drawn from the initial analyses of these data - that aspects inherent to the illness itself contribute both to delayed access to treatment and to poor outcome. ${ }^{7}$ Prolonged DUP has been associated with certain neurocognitive impairments at presentation ${ }^{33}$ and with grey matter changes, including reductions in planum temporale, ${ }^{34}$ left middle and inferior temporal lobe and occipital and fusiform cortices, and grey matter excess in left basal ganglia. ${ }^{35}$ The intriguing question is whether change of this sort reflects the action of a putative 'toxin' or whether it is itself the 'noxious' element, with structural brain abnormalities representing biological substrates for 'types' of illness whose clinical features inherently frustrate the aims of early intervention programmes.

\section{David Cunningham Owens, MD, FRCPsych, Eve C. Johnstone, MD, FRCPsych, Patrick Miller, PhD, Division of Psychiatry, University of Edinburgh, Royal Edinburgh Hospital, Edinburgh; J. Fiona Macmillan, MD, MRCPsych, South Staffordshire Healthcare Trust, Stafford; Timothy J. Crow, PhD, FRCPsych, Prince of Wales International Centre, Department of Psychiatry, University of Oxford, Warneford Hospital, Oxford, UK}

Correspondence: Professor David Cunningham Owens, Division of Psychiatry, University of Edinburgh, Kennedy Tower, Royal Edinburgh Hospital, Morningside Terrace, Edinburgh EH10 5HF, UK. Email: david.owens@ed.ac.uk

First received 24 Apr 2009, final revision 21 Nov 2009, accepted 2 Dec 2009

\section{Acknowledgements} The authors are grateful to all those colleagues who referred patients for this study, indi-
vidually acknowledged in the original publications, and to the patients and their families for their participation. Dr A.L. Johnson of the MRC Biostatistics Unit, Cambridge, performed the original analyses and Dr Andrew Mclntosh kindly advised on aspects of the re-analysis.

\section{References}

1 Wyatt RJ. Neuroleptics and the natural course of schizophrenia. Schizophr Bull 1991; 17: 325-51.

2 Marshall M, Lewis S, Lockwood A, Drake R, Jones P, Croudace T. Association between duration of untreated psychosis and outcome in cohorts of firstepisode patients. Arch Gen Psychiatry 2005; 62: 975-83.

3 Perkins D, Gu H, Boteva K, Lieberman J. Relationship between duration of untreated psychosis and outcome in first-episode schizophrenia: a critical review and meta-analysis. Am J Psychiatry 2005; 162: 1785-804.

4 Verdoux $\mathrm{H}$, Cougnard $\mathrm{A}$. The early detection and treatment controversy in schizophrenia research. Curr Opin Psychiatry 2003; 16: 175-9.

5 Warner R. Problems with early and very early intervention in psychosis. $\mathrm{Br} \mathrm{J}$ Psychiatry 2005; 187 (suppl 48): s104-7.

6 Verdoux H, Liraud F, Bergey C, Assens F, Abalan F, van Os J. Is the association between duration of untreated psychosis and outcome 
confounded? A two year follow-up study of first-admitted patients. Schizophr Res 2001; 49: 231-41.

7 Crow TJ, MacMillan JF, Johnson AL, Johnstone EC. The Northwick Park Study of first episodes of schizophrenia. II. A randomised controlled trial of prophylactic neuroleptic treatment. Br J Psychiatry 1986; 148: 120-7.

8 Johnstone EC, Crow TJ, Johnson AL, MacMillan JF. The Northwick Park Study of first episodes of schizophrenia. I. Presentation of the illness and problems relating to admission. Br J Psychiatry 1986; 148: 115-20.

9 MacMillan JF, Crow TJ, Johnson AL, Johnstone EC. The Northwick Park Study of first episodes of schizophrenia. III. Short-term outcome in trial entrants and trial eligible patients. Br J Psychiatry 1986; 148: 128-33.

10 Wing J, Cooper J, Sartorius N. The Description and Classification of Psychiatric Symptoms: An Instruction Manual for the PSE and CATEGO Systems. Cambridge University Press, 1974.

11 Jablensky A, Schwartz R, Tomov T. Collaborative study on impairments and disabilities associated with schizophrenic disorders. A preliminary communication. Objectives and methods. Acta Psychiatr Scand 1980; 62 (suppl 285): 152-63.

12 Cunningham Owens DG, Miller $P$, Lawrie SM, Johnstone EC. Pathogenesis of schizophrenia: a psychopathological perspective. Br J Psychiatry 2005; 186 386-93.

13 Lawrie S, Johnstone E. Schizophrenia and related disorders. In Companion to Psychiatric Studies (eds E Johnstone, D Cunningham Owens, S Lawrie, M Sharpe, C Freeman): 390-420. Churchill Livingstone, 2004

14 Craig T, Bromet E, Fennig S, Tanenberg-Karant M, Lavelle J, Galambos N. Is there an association between duration of untreated psychosis and 24-month clinical outcome in a first-admission series? Am J Psychiatry 2000; 157: 60-6.

15 Compton M, West J, Olfson M. Prolonged duration of untreated psychosis in non-affective first-episode psychotic disorders compared to other psychoses. Inter J Psychiatr Clin Pract 2007; 10: 264-8.

16 Johnstone EC, MacMillan JF, Frith CD, Benn DK, Crow TJ. Further investigation of the predictors of outcome following first schizophrenic episodes. Br J Psychiatry 1990; 157: 182-9.

17 American Psychiatric Association. Diagnostic and Statistical Manual of Mental Disorders (4th edn) (DSM-IV). APA, 1994.

18 World Health Organization. The ICD-10 Classification of Mental and Behavioural Disorders: Clinical Descriptions and Diagnostic Guidelines. WHO 1992

19 Barnes TRE, Hutton SB, Chapman MJ, Mutsatsa S, Puri BK, Joyce EM. West London first-episode study of schizophrenia. Clinical correlates of duration of untreated psychosis. Br J Psychiatry 2000; 177: 207-11.

20 Barnes TRE, Leeson VC, Mutsatsa SH, Watt HC, Hutton SB, Joyce EM. Duration of untreated psychosis and social function: 1-year follow-up study of first-episode schizophrenia. Br J Psychiatry 2008; 193: 203-9.
21 Norman R, Malla A, Manchanda R. Delay in treatment for psychosis: its relation to family history. Soc Psychiatr Psychiatric Epidemiol 2007; 42: 507-12.

22 Johnstone EC, Ebmeier KP, Miller $\mathrm{P}$, Owens DGC, Lawrie SM. Predicting schizophrenia: findings from the Edinburgh High-Risk Study. Br J Psychiatry 2005; 186: 18-25.

23 Drake RJ, Haley CJ, Akhtar S, Lewis SW. Causes and consequences of duration of untreated psychosis in schizophrenia. Br J Psychiatry 2000; 177: $511-5$.

24 Melle I, Larsen T, Haahr U, Friis S, Hohannesen J, Opjordsmoen S, et al. Prevention of negative symptom psychopathologies in first-episode schizophrenia Arch Gen Psychiatry 2008; 65: 634-40.

25 Owens D. How CATIE brought us back to Kansas: a critical reappraisal of the concept of atypical antipsychotics and their place in the treatment of schizophrenia. Adv Psychiatr Treat 2008; 14: 17-28.

26 Owens DG, Johnstone EC. The disabilities of chronic schizophrenia - their nature and the factors contributing to their development Br J Psychiatry 1980; 136: 384-95.

27 Morgan C, Abdul-Al R, Lappin JM, Jones P, Fearon P, Leese M, et al. Clinical and social determinants of duration of untreated psychosis in the AESOP first-episode psychosis study. Br J Psychiatry 2006; 189: 446-52.

28 Chow D, Law B, Chang E, Chan R, Law C, Chen E. Duration of untreated psychosis and clinical outcome 1 year after first-episode psychosis. Hong Kong J Psychiatry 2005; 15: 4-8.

29 Singh S. Outcome measures in early psychosis. Relevance of duration of untreated psychosis. Br J Psychiatry 2007; 191 (suppl): s58-63.

30 Schmitz N, Malla A, Norman R, Archie S, Zipursky R Inconsistency in the relationship between duration of untreated psychosis (DUP) and negative symptoms: sorting out the problem of heterogeneity. Schizophr Res 2007; 93: 152-9.

31 Norman R, Malla A, Manchanda R. Early premorbid adjustment as a moderator of the impact of duration of untreated psychosis. Schizophr Res 2007; 95: 111-4.

32 Wunderink A, Nienhuis F, Sytema S, Wiersma D. Treatment delay and response rate in first episode psychosis. Acta Psychiatr Scand 2006; 113: 332-9.

33 Lappin J, Morgan K, Morgan C, Dazzan P, Reichenberg A, Zanelli J, et al. Duration of untreated psychosis and neuropsychological function in first episode psychosis. Schizophr Res 2007; 95: 103-10.

34 Takahashi T, Suzuki M, Tanino R, Zhou S-Y, Hagino H, Niu L, et al. Volume reduction of the left planum temporale gray matter associated with long duration of untreated psychosis in schizophrenia: a preliminary report. Psychiatry Res: Neuroimag 2007; 154: 209-19.

35 Lappin J, Morgan K, Morgan C, Hutchison G, Chitnis X, Suckling J, et al. Gray matter abnormalities associated with duration of untreated psychosis. Schizophr Res 2006; 83: 145-53. 